\title{
Chronisch-entzündliche Hauterkrankungen
}

Michael Sticherling

Hautklinik, Universitätsklinikum Erlangen, Erlangen, Deutschland

\section{Hintergrund}

Chronisch-entzündliche Dermatosen zählen zu den häufigsten Hauterkrankungen [1]. Betroffen sein können dabei einzelne oder alle drei Hautschichten Epidermis, Dermis und Subkutis [2]. Klassische Zeichen sind die entzündliche Rötung, die jedoch abhängig von der Lokalisation unterschiedlich sein kann, und vielfach auch eine mehr oder weniger deutliche Schuppung. Wichtig ist, bei der Diagnose zu unterscheiden, ob es sich um eine exogen oder um eine endogen ausgelöste Erkrankung handelt [2].

Häufige Krankheitsbilder und ihre Therapieoptionen

\section{Atopische Dermatitis (Neurodermitis)}

Die Ätiopathogenese der Neurodermitis ist bis heute noch nicht vollständig geklärt. Als mögliche Auslöser gelten u.a. Dysregulation des Hautimmunsystems, Störungen des neurovegetativen Systems sowie Anomalien der epidermalen Hornschicht [3], aber auch genetische Aspekte. Therapeutisch ist zunächst eine konsequente Rückfettung der Haut wichtig. Abhängig von der Akuität der Erkrankung wird eine topische antientzündliche Behandlung mit Kortikosteroiden oder Calcineurin-Inhibitoren, gegebenenfalls in Kombination mit ultraviolettem Licht, bei schweren Krankheitsbildern auch eine Systemtherapie mit Immunsuppressiva und Immunmodulatoren eingesetzt. Seit kurzem steht ein effektiver und gut verträglicher humaner Antikörper (Biologikum) zur Verfügung, der spezifisch den pathogenetisch bedeutsamen Interleukin(IL)4-Rezeptor blockiert.

\section{Kollagenosen (entzündliche Bindegewebserkrankungen)}

Diese Gruppe von Erkrankungen zeichnet sich durch entzündliche, vermutlich autoimmun vermittelte Veränderungen an den dermalen Gefäßen und dem Bindegewebe aus. Neben der Haut können zahlreiche innere Organe einbezogen sein. Die Ätiopathogenese der Erkrankungen ist bis heute nicht detailliert geklärt, diagnostisch lassen sich jedoch häufig spezifische Autoantikörper nachweisen [4]. Die Therapie richtet sich nach Akuität und Progress der Erkrankung sowie Ausmaß der Organbeteiligung und umfasst in der Regel systemische immunsuppressive und immunmodulatorische Substanzen.

\section{Lichen planus (Knötchenflechte)}

Es ist eine häufige Hauterkrankung mit stark juckenden, bläulichroten Papeln und Plaques, die vor allem bei Erwachsenen auftritt - dabei sind Männer häufiger als Frauen betroffen. Vermutet wird u.a. eine virusbedingte Autoimmunreaktion, aber auch kontaktallergische Reaktionen [5]. Bei den umschriebenen Veränderungen sind topische Kortikosteroide, gegebenenfalls in Kombination mit UV-Licht, bei schweren Krankheitsbildern eine immunsuppressive Systemtherapie erforderlich.

\section{Psoriasis (Schuppenflechte)}

Erhabene, unregelmäßig begrenzte, rötliche Plaques der Haut mit silbriger Schuppung weisen auf diese Erkrankung hin [6]. Vor einem immungenetischen Hintergrund können verschiedene innerliche und äußerliche Faktoren die Erkrankung auslösen [7]. Neben der Haut können Gelenke und Sehnen betroffen sein (PsoriasisArthritis) sowie metabolische und kardiovaskuläre Begleiterkrankungen das Krankheitsbild verkomplizieren. Abhängig vom Schweregrad der Erkrankung ist eine topische, UV- oder Systemtherapie erforderlich. Ende 2017 ist dazu die überarbeitete S3Leitlinie erschienen [8].

\section{Rosazea}

Sie zeigt sich in verschiedenen Stadien mit initial flushartigen, später dann persistierenden Erythemen und Teleangiektasien sowie entzündlichen Episoden mit Papeln und Pusteln im Gesicht. Hauptmanifestationsalter liegt zwischen dem 40. und 60. Lebensjahr. Entzündliche Vorgänge im Bereich der dermalen Gefäße, möglicherweise eine Fehlbesiedlung der Haut und eine Störung der angeborenen Immunität sind pathogenetisch bedeutsam. Abhängig von Lokalisation und Akuität der Erkrankung wird eine topische oder systemische Therapie durchgeführt, wobei die häufig eingesetzten Antibiotika vermutlich eher eine immunmodulatorische als eine antibaktierelle Wirkung haben.

\section{KARGER \\ Fax +497614520714 \\ information@karger.com}

www.karger.com
() 2018 S. Karger GmbH, Freiburg

Accessible online at:

www.karger.com/kkd
Prof. Dr. Michael Sticherling

Hautklinik, Universitätsklinikum Erlangen,

Ulmenweg 18, 91054 Erlangen, Deutschland

michael.sticherling@uk-erlangen.de 
Entzündliche Erkrankungen machen einen großen Teil der Hauterkrankungen aus. Sie müssen differentialdiagnostisch abgeklärt und je nach Schwere der Erkrankung behandelt werden. Für viele stehen heute effektive und gut verträgliche topische wie systemische Präparate zur Verfügung. Um die Chronifizierung (z.B. atopisches Ekzem), Narbenbildung (z.B. diskoider Lupus erythematodes) oder Folgen von Begleiterkrankungen (z.B. Psoriasis) zu verhindern, sollten bei mittelschweren und schweren Krankheitsbildern rechtzeitig eine Systemtherapie eingeleitet und die Patienten konsequent beobachtet werden. Aspekte der Lebensführung wie auch Lebensqualität sind individuell zu berücksichtigen.
1 Schmid C: Weitere entzündliche Dermatosen; in Goebeler M, Hamm H (Hrsg.): Basiswissen Dermatologie, ed 1 Berlin, Springer, 2017, p 93.

2 Arbeitsgemeinschaft Dermatologische Prävention: Dermatosen. www.unserehaut.de/de/unsere-haut/Dermatosen.php. (Zugriff: 12.03.2018),

3 DocCheck Flexikon: Neurodermitis. http://flexikon.doccheck.com/de/ Neurodermitis. (Zugriff: 12.03.2018)

4 DocCheck Flexikon: Kollagenosen. http://flexikon.doccheck.com/del Kollagenose. (Zugriff: 12.03.2018).

5 DocCheck Flexikon: Lichen planus. http://flexikon.doccheck.com/de/Lichen_ ruber_planus. (Zugriff: 12.03.2018).

6 DocCheck Flexikon: Psoriasis vulgaris. http://flexikon.doccheck.com/de/ Psoriasis_vulgaris. (Zugriff: 12.03.2018).

7 Mrowietz U; Schmidt-Ott G: Schuppenflechte, ed 4 Freiburg, Karger, 2017. p 6.

8 Nast A, Amelunxen L, Augustin M, et al.: S3-Leitlinie zur Therapie der Psoriasis vulgaris. AWMF Register-Nummer 013-001. Stand 10/2017. www.awmf.org/ uploads/tx_szleitlinien/013-001I_S3_Therapie_Psoriasis-vulgaris_2017-12.pdf. (Zugriff: 12.03.2018) 